\title{
Preparation of Thin Sections
}

\author{
Ian Chaplin, Buehler Krautkramer
}

\section{It is no longer labour intensive, but still requires knowledge} and care.

The optical examination of a rock sample in thin section is the quickest and most economical method for classifying rock type and determining which analytical route to follow.

Thin sections for transmitted light are the most common, but there are also:

Polished Thin Sections - Polished sections are used for classification and identification of minerals that cannot be determined in standard thin sections. They are also essential for microprobe analysis. Minute mineral grains are analyzed by bombarding them with a focused bean of electrons, which generate $x$-rays, characteristic of the elements within the grains. X-rays are identified and quantified to determine the chemical composition of minerals.

Double-sided Polished Thin Sections (Ultra-thin) - Samples of this nature are normally analyzed on a transmission electron microscope at a thickness of 1-2 $\mathrm{um}$ Crystal structure cannot be determined at $30 \mu \mathrm{m}$ in samples like meteorites, coals and shales.

Fluid Inclusion Wafers - These samples are normally analyzed using a microscope fitted with a heating, freezing stage with a temperature range of $-200^{\circ} \mathrm{C}$ to $+600^{\circ} \mathrm{C}$. By analyzing the fluid/gas bubble one can determine the age and style of mineralisation.

Polished Specimens - This method is used on samples containing opaques and ores where hardness testing and reflectivity are used to identify minerals. Reflected light microscopy is used for viewing this particular type of sample.

\section{Preparation of Thin Sections}

Thin section preparation consists of thinning mounted rock or mineral samples to a thickness of approximately $30 \mu \mathrm{m}$. Successful preparation of thin sections requires considerable manual skill or the use of precision equipment to maintain the required control of thickness and planoparallelism. It is normally not necessary to

\section{Take the following microanalysis quiz}

\author{
What is the thickness of my film? \\ Does the beam penetrate that particle? \\ What is the best $k V$ to use for this sample? \\ How wide is the beam in my E-SEM? \\ How much does an incorrect analysis cost? \\ How can I improve the quality of my analysis?

\section{Maybe it's time to take a look at the software that can answer these questions}

\section{Electron Flight Simulator \\ Analysis Simulation and Modeling Software for Windows}

\section{Small World}

phone/fax: (703) 849-1492

e-mail: dchernoff@aol.com http://www.small-world.net produce a scratch-free polished surface.

Many years ago, thin sections were prepared entirely by hand. Hand lapping was carried out on a glass slide, using a series of abrasive powders of decreasing size. The lapped surface was then mounted on a glass microscope slide using a natural mounting medium (Canada Balsam). The other surface was then ground on a glass plate, again using a series of abrasives, until the desired thickness of $30 \mu \mathrm{m}$ was achieved.

Today most of these stages are carried out using sophisticated pieces of equipment, some of which are automated.

Thin sectioning consists of a series of preparation steps:

1. Sectioning (taking initial slice from specimen)

2. Vacuum or Pressure Impregnation (where necessary)

3. Trimming (cutting slice to size)

4. Lapping (removal of deformed surface created by initial slice cutting)

5. Bonding lapped surface of rock slice to glass

6. Resectioning (removing excess material)

7. Finishing (final stages of sample preparation)

8. Coverslipping (putting the protective slip over thin section)

\section{Sectioning}

Fix the rock specimen to be sectioned in the clamp of the trim saw, making sure the sample is held firm. Take first cut through the specimen to produce a flat surface. Then move the sample $3-4 \mathrm{~mm}$. A thickness of $3-4 \mathrm{~mm}$ combines the strength necessary for handling with specimen economy. Take a second cut to produce a parallel slice. Remove rock from machine clamp, dry the slice and label it with a preparation number

\section{Vacuum Impregantion}

Vacuum impregnation, used with porous or friable materials, is the process of evacuating air and other gasses from pores or cracks and replacing them with a suitable bonding material such as epoxy resin. The cured resin will protect the sample from damage, such as fracturing or plucking, by entering and filling all cracks, voids and pores. This also provides an uninterrupted surface that will not trap abrasive swarf of liquids which could cause contamination and stains.

Geological samples for top surface polishing are vacuum impregnated as a matter of course and petroleum geologists will often infiltrate with a colored resin to help determine porosity.

Pressure impregnation is similar, but uses high pressure to force the resin between the pores.

\section{Trimming}

Place the sample flat on the worktable and with a constant pressure, push the slice under the wheel guard. This is normally done four times to produce a trimmed slice of suitable size to fit glass slide/coverslip to be used.

A glass coverslip, glass microscope slide or a go-no-go gauge can be used as a guide to size. Do not cut off the sample identification number!

\section{Lapping}

Lapping the sample can be carried out by hand or with a lapping machine, such as the Buehler Krautkramer Petrolap.

Lapping by Hand - First mix a paste on the glass plate using a suitable sized abrasive, such as 400 grit silicon carbide and water (all grit sizes referred to in this guide are FEPA grades). Place the slice down on the glass plate making sure the number is on the upper surface, hold the slice at each corner between thumb and index finger and rotate specimen in small loops utilizing all of the glass plate. Keep changing the position of the slice and replenish with more silicon carbide and water as the paste thins or dries out. After continuous lapping for approximately two to three minutes, before checking the lapped surface, thoroughly scrub and dry the slice. This can be done by reflecting the light from a strip light directly from the slice surface into the eyes. Surface irregularities and quality of surface finish can be determined by checking to see if any major unevenness or cut marks are apparent. Lapping on $\mathbf{4 0 0}$ grit silicon carbide should be continued until the surface has a completely even matt appearance.

Proceed to new glass plate with 600 grit silicon carbide using the same procedure as with the 400 grit. Check surface.

Progress to 1000 grit silicon carbide on a separate glass plate, as previously. done The fine grade of silicon carbide provides better grain edge definition, improves grain integrity and grain flatness, therefore enhancing image quality under the microscope. The sample looks polished when light is reflected off the surface.

After each of the lapping stages the sample should be cleaned thoroughly. An ultrasonic cleaner will give best results.

Machine Lapping - Load the rock slices into conditioning rings with numbered side up. Fill rings to maximum capacity using small pieces of scrap rock as necessary. Place a 
sponge pad on top of the specimens, followed by the weight. Lap for 30 minutes using 600 grit silicon carbide dispensed from a suitable abrasive slurry feed system. Lapping with a machine such as the Buehler Krautkramer Petrolap leaves the operator free to prepare more samples for lapping or to continue with other stages in the thin section process.

Glass slides can be used frosted (lapped) or unfrosted depending on requirements or machines used. Frosted surfaces, however, give a better bond between slice and glass slide. Polished glass slides can be frosted on a glass plate using 600 grit silicon carbide and water, as used in the rock slice lapping process. Slides intended for automatic preparation should be pre-ground on specific machines to the required thickness, this being essential for a machine finishing process.

Write sample identification number on the reverse side of the glass slide.

\section{Bonding}

Consists of attaching flat lapped rock slides to glass microscope slides using Lakeside 70, Epoxy Resin or UV sensitive adhesive.

\section{Resectioning and Grinding}

Resectioning is used in thin section preparation for two reasons: 1. It leaves you with a spare slice of the rock which can be re-used.

2. It is quicker to cut off $2-3 \mathrm{~mm}$ of rock than to grind it off.

The resectioning thickness varies depending on rock type.

\section{Machine Grinding}

The Petrothin Thin-Sectioning System from Buehler Krautkramer will resection and fine grind thin sections of all types of rocks and non-metallics to a thickness of approximately $45-80 \mu \mathrm{m}$. The thickness will vary depending upon the materials being sectioned. It is recommended that finish lapping of the section be accomplished by hand in a flat glass plate charged with aluminum oxide or silicon carbide. This final hand polishing procedure reduces the possibility of fracturing or inducing structural damage in the final stages of preparation.

\section{Hand finishing}

Finish by hand using 600 grit abrasive on a glass plate. Mix paste and lap the sec- tion to approximately $40 \mu \mathrm{m}$. Frequent examination with either the polarized-light microscope or a viewer such as the Buehler Krautkramer Petrovue is essential during this critical process in thin section preparation to monitor progress and ensure the slice is totally parallel throughout its length. When the slice has reached $40 \mu \mathrm{m}$ (quarz first order yellow), throughly wash both hands and the slice.

The last $10 \mu \mathrm{m}$ are removed by lapping the slice on grade 800 or 1000 grit abrasive. The lapping procedure is the same as that used with the 600 grit abrasive.

Sufficient pressure must be applied to the slice for the abrasive action to be efficient, but care must be taken if the slice is not to be ground away entirely. Frequent checks on progress using a polarized-light microscope are required. Normally, sections are ground to a final thickens of $30 \mu \mathrm{m}$ (quartz first order white, or very pale yellow).

As the section is ground the interference colors will change with from the purples and reds through to oranges and yellows. Grinding should continue until all the quart crystals show only grays and whites when viewed with crossed polars.

\section{Coverslipping}

On completion of final lapping of the thin section, it is necessary to add a protective coverslip. This prevents deterioration of the surface and its constituents. It also improves the microscopic image by filling pits etc. with mounting media.

NOTE: The thin section preparation described does not lend itself to all materials to be sectioned. Preparation routes are dictated by the materials to be prepared and anabyzed..

\section{ACKNOWLEDGEMENTS:}

The author would like to acknowledge the assistance of Dr A. G. Tindle of the Department of Earth Sciences, Open University, for his general assistance with this article and discussions. Parts of this article are based on the work of W Ahmed (Petrographic sample preparation for microstructural analysis Buehler Digest $24(1))$ and the section on hand lapping and hand finishing is based on Allman \& Lawrence (1972) General Geological Technique Blandford Press, London.

This article originally appeared in Microscopy And Imaging News (UK), February 1998 issue.

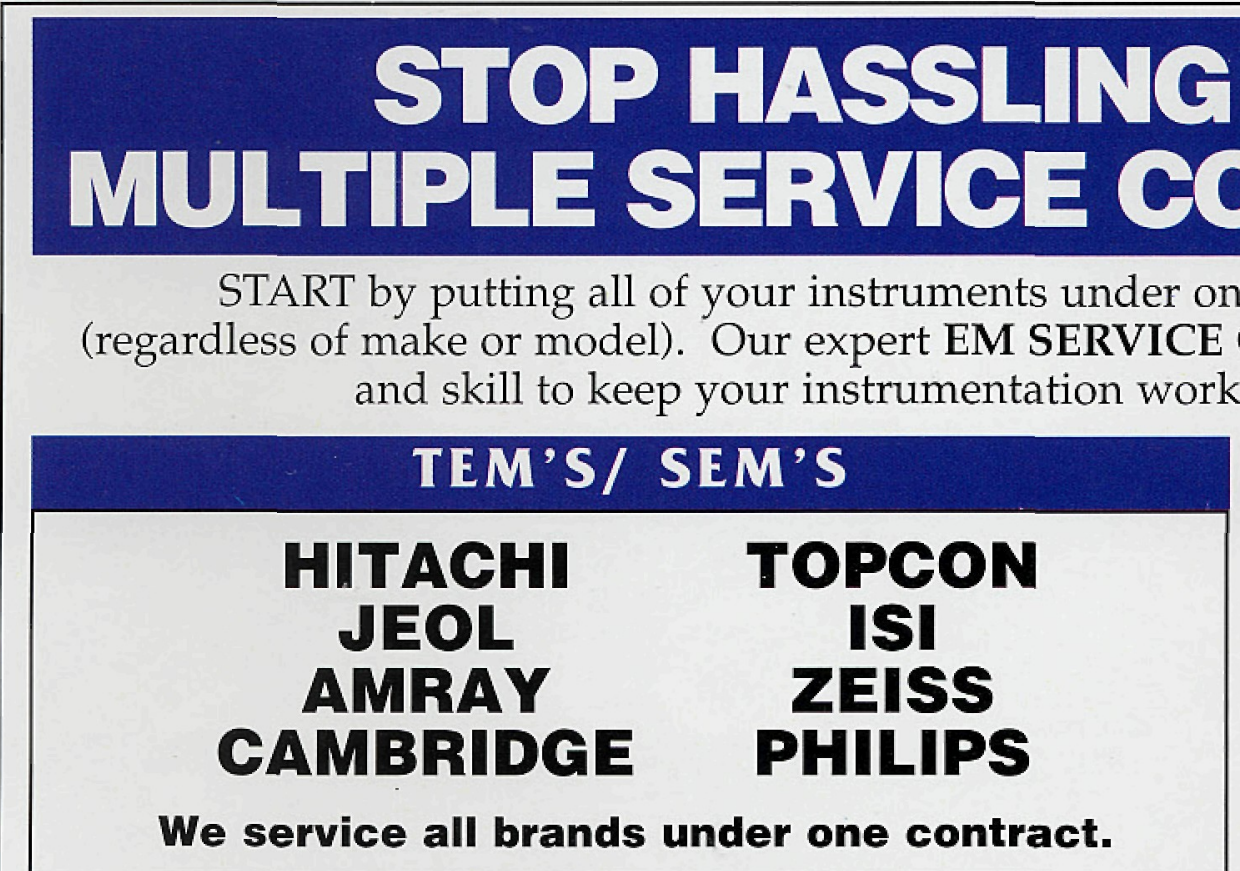

We service all brands under one contract.

WE SERVICE COMPUTER CONTROLLERS

BEAM BLANKERS WATER CHILLERS
Contracts and On-Demand

Emergency Service at

Reasonable Rates from

Factory Trained Specialists.

\section{1-800-421-8451}

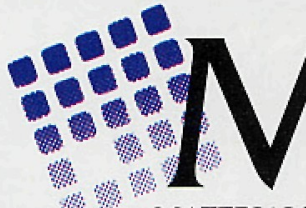

MATERIALS ANALYTICAL SERVICES

3597 Parkway Lane • Suite 250 • Norcross, Georgia 30092 • 770-448-3200 • FAX 770-368-8256 or 616 Hutton Street - Suite 101 - Raleigh, North Carolina 27606 • 919-829-7041 • FAX 919-829-5518 ADVANCED ANALYTICAL PRODUCTS AND SERVICES 